\title{
Multi-Agent Based Cargo Auction
}

\author{
Egons Lavendelis ${ }^{1}$, Janis Grundspenkis ${ }^{2}$, \\ ${ }^{1,2}$ Riga Technical University, Latvia
}

\begin{abstract}
The paper presents a mechanism and an implemented tool for multi-agent based cargo auctions. The auction automates negotiations between a particular client and multiple logistics companies. The user can define his priorities in terms of six different criteria and find the most appropriate offer as a result of an auction. The developed marketplace is part of the eINTERASIA e-logistics portal and, thus, available online for both the client and the logistics companies.
\end{abstract}

Keywords - Auction protocols, automated marketplace, cargo transportation and logistics, multi-agent negotiation mechanisms, multi-agent system.

\section{INTRODUCTION}

Nowadays many markets involve large numbers of buyers and sellers. Each buyer is willing to find the most appropriate seller for his needs. The buyers can be either companies or private clients. Small companies and private clients cannot afford to invest in any automated IT solutions for finding the most appropriate seller and offer. Usual approach is the following. The sellers make their offers available online and clients access the offers of various companies online and sometimes even make orders online. Still, the comparison between different companies is manual in many markets, including cargo transportation and logistics domain. Additionally, the strategic question of the pricing that the seller company should offer to its clients is complex and many resources are spent on market studies done at each seller company with the aim to get a competitive advantage over other sellers. To sum up, one of the problems in today's markets is to automatically get the price from different suppliers. Some price comparison solutions exist for internet shops, for example, Latvian portals salidzini.lv and kurpirkt.lv. Still they do not allow the sellers to vary the price or the offered service in different situations as well as such portals usually exist just for internet shops selling finite and rather small sets of products instead of services that should be adapted to each particular client. In particular, these price comparison portals can just compare prices for static offers without giving any possibilities for the suppliers to create individualized solutions for each client.

One possibility to save effort on the market studies at the companies as well as clients' time to choose the most appropriate solution for his needs is to implement an automated electronic marketplace where the companies can offer their services and clients can automatically find the most appropriate offers for themselves. Because of the differences in the criteria and models used by clients to evaluate offers, a unique or customized solution is needed for each individual market. An example of such a marketplace exists for the travel insurance policy domain [1] where clients can enter their preferences into the market automation software and get offers from the companies participating in the market. Still the tool is not available online and it is just a simulation tool that can be installed on a particular computer.

The paper describes a tool that demonstrates automation of a cargo transportation market and creates a marketplace for buying and selling cargo logistics solutions. The tool is based on a multi-agent system within which agents carry out an auction where each order is auctioned among the possible suppliers and the client can get the most appropriate offer according to the chosen criteria and importance of each criterion.

The remainder of the paper is organized as follows. Section II defines the main requirements for the marketplace automation solution. Section III describes the developed multiagent system. Section IV defines the used mathematical model for the offer evaluation. Section $\mathrm{V}$ describes the developed auction tool for marketplace automation. Section VI outlines the access to the tool by its administrator. Section VII concludes the paper and outlines the future work.

\section{REQUIREMENTS FOR THE MARKETPLACE}

The aim of the research is to create a marketplace that would automate the interactions between the logistics companies that offer cargo transportation from Asia to Europe and their clients that need the actual transportation to be done. The client knows the location of the cargo and the needed destination as well as may have some preferences in terms of characteristics of the transportation process like time, security, etc.

The first requirement for the marketplace is non-functional. The marketplace should be accessible online both by the clients to find the most appropriate deal and by the logistics companies that offer the cargo transportation services.

To help the companies in solving the problem of finding and executing the best strategic behavior of the company and implementing it into the automated marketplace, each company should be represented by an autonomous software component that may differ from other companies by the strategy that it uses to represent the corresponding company. These autonomous entities should be capable to communicate and negotiate among themselves to automatically find the deal (without any human actions between the input of the criteria and the output of the most appropriate deal) that is presented to the potential user. Autonomous representation of stakeholders at the marketplace in the latest studies is achieved by the use of intelligent agent paradigm [2]-[4]. In these studies, marketplaces are built so that each stakeholder is represented by an intelligent autonomous agent. In the case of cargo transportation, it means that each 
logistics company is represented by its agent as well as the client is represented by his agent. The essential benefit of using intelligent agents is their capability to act autonomously and represent their owners by doing proactive actions to achieve the user's goals. These agents are chosen to be the autonomous software components that implement the particular market strategies of each company. The intelligent agents and the multi-agent system built from them to implement the automatic cargo marketplace are described in Section III.

The third major requirement is the possibility to find the most appropriate transportation deal by more than one criterion, because the cheapest solution is not always the best one and, thus, the price is not the only criterion to consider. Criteria such as price, delivery time, security, possibility to access the data about the current location of the cargo, reputation of the company are among the most important criteria to consider. In general, there might be more criteria that are important for the client and, thus, the model used to evaluate deals should be extendable with additional criteria. The current model used to evaluate the deal by multiple criteria is outlined in Section IV.

Lastly, a negotiation protocol is needed to implement automated negotiations between the client agent and the company agents. The general multi-agent negotiation protocols for the deals with multiple attributes or criteria are usually based on multiple negotiation rounds and the notion of concession and, as a consequence, require complex game theory based strategic deliberations to find the optimal individual strategies [5]. This would lead to the increase of the investments in the market research and finding the best strategy. Thus, the requirement is that the negotiation protocol used at the marketplace is simple in terms of finding the optimal strategy.

To sum up, the main requirements of the electronic marketplace for the transportation domain are the following:

- The marketplace is available to the client and logistics companies online;

- The client and the logistics companies are represented by the autonomous software components;

- The autonomous software components representing the logistics companies should implement the corresponding company's market strategy;

- The negotiations among the client and the logistics companies must be automatic and there should be no human involvement between the choice of the criteria and the output of the most appropriate deal that is available at the marketplace;

- Multiple criteria should be used to find the most appropriate deal and the model should be open for new criteria. The values of all criteria that are important for the client must contribute to the utility of each deal during the process of evaluation of deals and choosing the best one;

- The negotiation protocol must be simple in terms of the choice of the strategy and should not require complex market research to find the most appropriate strategy.

\section{THE Multi-AgENT SYSTEM}

As discussed in Section II, the marketplace is implemented by a multi-agent system. Each logistics company as well as the client is represented by particular autonomous agents. The agents are named a client agent and a company agent, respectively.

As concluded in Section II, there is a need for the negotiation mechanism between the client agent and the company agents with simple individual strategies. According to [5], multi-agent auction mechanisms are simple and effective and, as a consequence, one of the most explored and applied negotiation mechanisms for one-to-many negotiations. Auction mechanisms have been used in the logistics domain to develop an offline electronic marketplace simulator [6]-[7], in the insurance domain to build a travel insurance policy market [1] as well as in labor management [8].

To use the auction as a negotiation mechanism, some commonly known evaluation model is needed to find the utility of deal for the client agent that is negotiating with logistics company agents for the cargo transportation deal. The traditional auctions are based on one criterion or one attribute of the deal, which in most cases is price [5], [7]. The cargo marketplace requires multiple criteria. Several auction approaches have been developed for such a purpose [6], [9], [10]. The approach used in the developed tool is outlined in Section IV.

To fit the requirements of the cargo transportation, marketplace traditional auction protocols with the following two extensions are used. First, instead of the price the deal must be evaluated by the weighted sum of all the criteria that are important for the client. Second, the auction is reverse in terms of the roles. The client's agent is the auctioneer that tries to maximize the utility of the deal (minimize the price and maximize other criteria), while the bidders are the company agents that try to maximize their income. Thus, the value of the utility is moving in reverse order compared to the traditional auctions. Still, this does not change the main mechanisms of the auction protocols and the traditional protocols can be adapted and used.

The four best known and widely used auction protocols according to [5] have been implemented. The client can choose the protocol to use in each particular case. The auction protocols are implemented in the following way:

- The English auction is an ascending open-cry auction. The client agent as an auctioneer starts the auction by announcing a new auction and giving weights defining the importance of all criteria as well as the initial value of the utility that is the minimum acceptable value for the client. The bidders can respond by submitting a bid. The response can be either a transportation offer or refusal to participate in the auction. After receiving an offer, the following offers must have a better utility for the client agent than the previous ones. The auction ends when no agent is willing to submit any bid with a better utility as the current bid has.

- The Dutch auction is an open-cry descending auction. The client agent starts the auction by announcing the weights 
of all criteria and the starting bid with an artificially high utility for the client. If no company agent is willing to propose a deal with the same utility, the client agent increases the price (so decreasing the utility) and asks for new bids. The client agent continues to increase the price until one of the company agents gives a bid with at least the same utility.

- The first-price sealed-bid auction starts with the client agent announcing the weights of all criteria. There is only one round of bidding and each agent is allowed to submit one offer. Offers are not visible to other participants. After all participants have submitted their offers (or the deadline has come), the auctioneer chooses the offer with the best utility.

- The Vickrey auction similarly to the first-price sealed-bid auction has only one round of bidding. The only difference is that it is a second price auction and in case of multiple criteria it is implemented in the following way. The utility of the winning deal is changed to be the same as the utility of the second best offer. It is done by increasing the price.

The structure of the developed multi-agent system is the following. It consists of 2 types of agents, namely, a single client agent and multiple company agents that represent the stakeholders of the cargo transportation market and participate in the auction protocol. The structure of the multi-agent system defining the roles of agents and showing interactions among agents is given in Fig. 1.

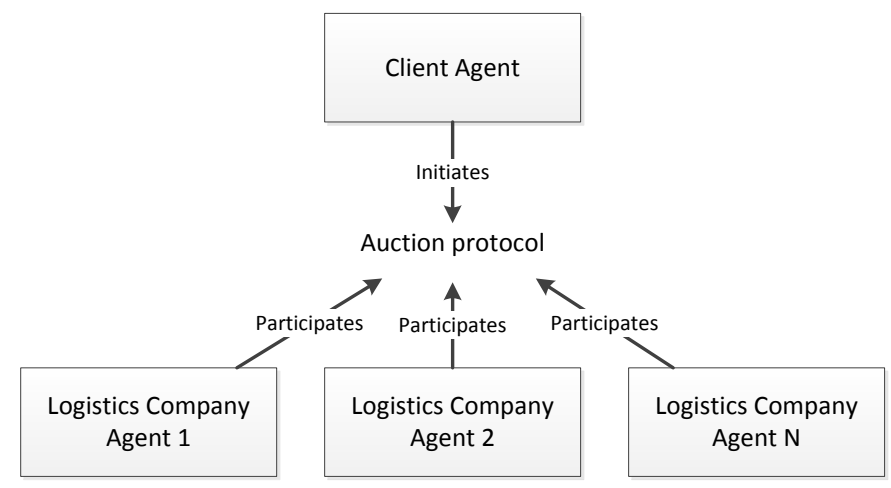

Fig. 1. Interactions among agents at the cargo transportation marketplace.

The extended version of the MASITS agent oriented software engineering methodology [11] was used for the development process of the multi-agent system based on the defined types of the agents.

\section{The Evaluation Model}

Although the price usually is the most important criterion used to make a choice among different cargo transportation options, it is not the only one. Various types of cargo may require different criteria. The paper will reuse the set of criteria that were found as the most important ones for the cargo transportation from Asia to Europe in [6]:

- Price, measured in currency per unit of the cargo;

- Transportation time, measured in minutes;
- Safety, measured in percent stating the probability to receive the cargo undamaged. It is based on the previous experience in the used transportation routes;

- Monitoring. The possibility to follow the location and status of the cargo. The scale is Boolean;

- Reliability. The client's subjective evaluation of the logistics company is based on the previous experience with the company. The scale is from 1 (very low) to 10 (very high);

- Reputation of the company as the partner. The scale is from 1 (very low) to 10 (very high). This criterion is subjective and varies among the clients.

The last two criteria are subjective and cannot be calculated by the logistics company. Thus, they must be added to the value of the utility by the client agent. The authors of [6] have created the following model to deal with the situation where values of some criteria are known only to the client agent, while other values are known only to the logistics company agents. The utility function of cargo transportation deals is defined by the following equation:

$$
U=\sum_{i=1}^{n} w_{i} * v_{i}, \text { where }
$$

$U$ - utility of the deal;

$n$ - number of criteria;

$w_{i}-i^{\text {th }}$ weight;

$v_{i}-$ value of $i^{\text {th }}$ criterion.

The company agent does not know subjective criteria that are just client's evaluations. As a consequence, the company agent can calculate only one part, named objective part of the utility function. The other part, namely, subjective part must be specified by the client agent. The model considers that the objective criteria are the first ones and the objective and subjective parts of the utility function can be calculated by Equations (2) and (3).

$$
\begin{gathered}
U_{o}=\sum_{i=1}^{n_{o}} w_{i} * v_{i}, \\
U_{s}=\sum_{i=n_{o}+1}^{n} w_{i} * v_{i}, \text { where }
\end{gathered}
$$

$U_{o}$ - objective part of the utility function;

$U_{s}$ - subjective part of utility function;

$n_{o}-$ number of known (objective) criteria for the company agent;

$n$ - the total number of criteria.

In open-cry auctions (English and Dutch), the company agents may decide to make a bid with a particular utility. The utility is adjusted by increasing the price. The actual value of the price that will give the needed value of the utility is calculated by using Equation (4) (assuming that the price is the first criterion):

$$
v_{1}=\frac{U-U_{S}-\sum_{i=2}^{n_{o}} w_{i} * v_{i}}{w_{1}}, \text { where }
$$

$v_{1}$ - price;

$w_{1}-$ weight of the price. 
The used model is general enough to allow adding any criterion to one of the groups - objective criteria or subjective criteria and so including it in the model. The model is reused in the developed auction platform.

\section{THE AUCTION PLATFORM}

The auction platform has been implemented to make the electronic marketplace available online. Currently the marketplace with test data from test companies is available online at the eINTERASIA web portal.

The developed tool automates the cargo transportation market from Asia to Europe in the following way. The client can use the developed auction platform to find the best deal according to the evaluation model outlined in the previous section. To find the offer the client has to provide the following inputs (see the top right part of the tool interface shown in Fig. 2):

- To specify the route in terms of start terminal and destination terminal. The terminals are chosen from the list that comes from the tool database. For the administration details see Section VI. The currently available terminals are also denoted in the map shown to the client in the tool main window (see Fig. 2).

- To choose the type of the auction. The client is allowed to choose among four types of auctions outlined in Section III.

- To specify the criteria that are important in the choice of the offer.

The following form of criteria input is used. For each criterion the weight and the initial value are specified. The initial value is meaningful only in English and Dutch auctions where it is needed to have the initial utility value to start the auction. Clients should remember that in the English auction the initial values define the worst possible deal that the client is ready to accept, while in the Dutch auction it defines an artificially good deal for the client and it is used to start the auction. The weights of the criteria are specified in the following form. Price always has the weight of 100 that allows specifying the other weights in the form of how many cents is the client ready to pay for each unit of the criterion. This method enables a precise definition of semantics of the measurement units used for the weights.

After the client starts the auction, his agent does the negotiations with the logistics company agents automatically according to the chosen auction protocol. The result of the auction is shown to the client in two ways. Firstly, the details of the result are outlined in the table at the bottom left part of the tool interface (see Fig. 2). The values of all 6 criteria and the name of the logistics company are given as well as the route is specified by the terminals, terminal operators and the transportation type used in each step of the route. Secondly, the route is visually drawn on the map (straight lines are used to denote the transportation from one terminal to another independently from the actual route).

See Fig. 2 for an example of the offer visualization. After receiving the offer if the client is satisfied with its details, he can choose to accept the deal by clicking the corresponding button.

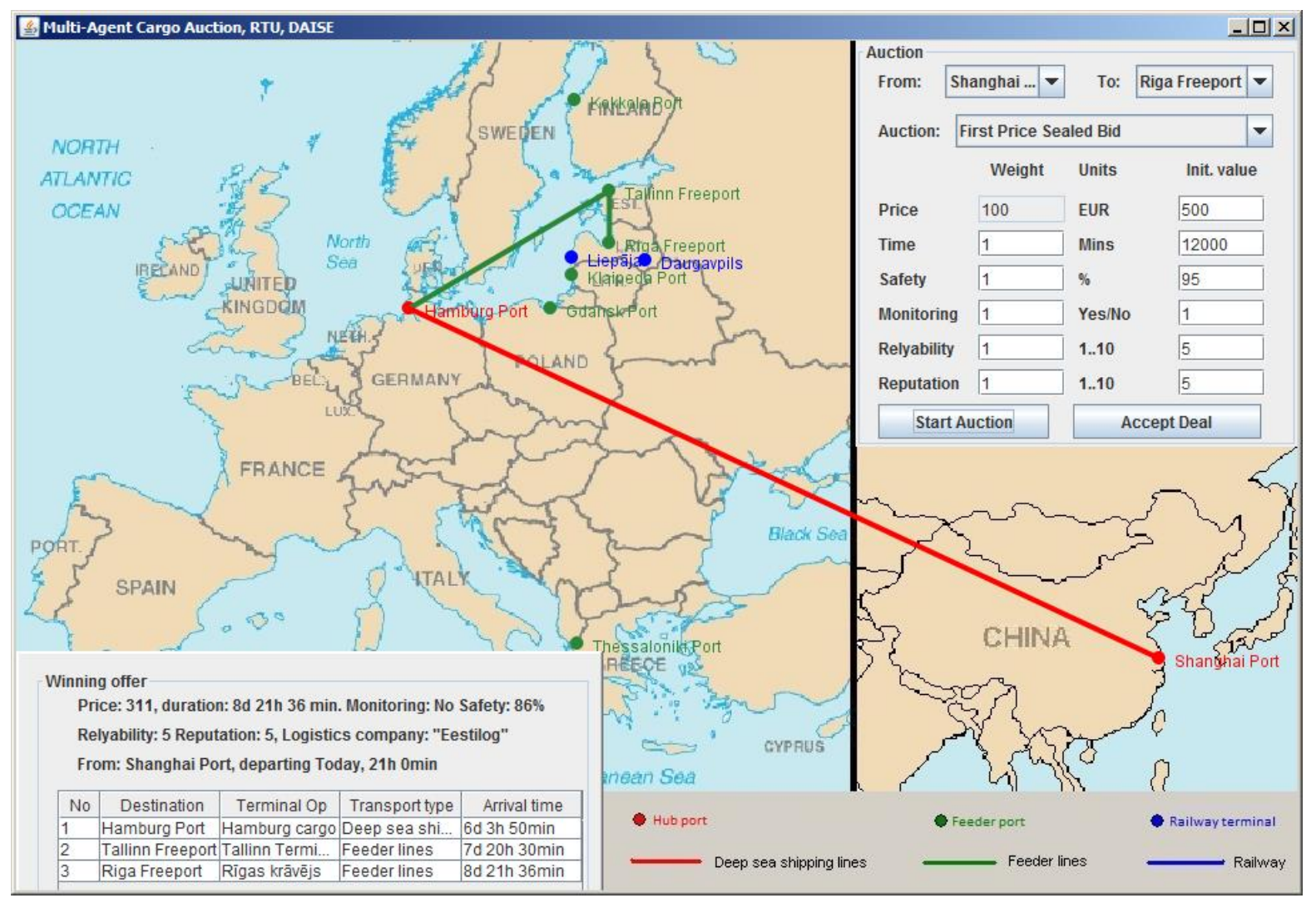

Fig. 2. The interface of the cargo auction tool with the visualization of the winning offer. 
The following two requirements were specified for the auction tool in Section II. Firstly, the tool must be available online. Secondly, the use of the autonomous agents as a core technology is followed from the fact that each stakeholder has to be represented by its own autonomous software component. To the author's knowledge the state-of-the-art agent development platforms do not provide a convenient way to deploy agents that represent stakeholders directly in the web environment, but only as Java processes. To fulfil both of these requirements, the auction platform has been developed using the JADE agent development framework [11] and Java language [12]. A web start has been created to integrate the tool into the eINTERASIA e-logistics portal and make it available online.

\section{ADMINISTRATOR'S ACCESS}

Additionally to the client's interface that is developed for the client to communicate with his agent, there is a need for the logistics companies to access the database and edit their offers. Currently the companies can access the tool database through the administrator's tool that is available in the restricted area of the eINTERASIA portal. The tool allows editing the available logistics companies, terminals, operators, carriers, schedules as well as the collaborations among the logistics companies, terminal operators and carrier companies. Simple forms for inserting, editing and deleting information from a database are available from the administrator's tool. An example of such a form is given in Fig. 3.

Currently the company agents are created automatically upon the start-up of the marketplace based on the information available in the database. Still, the information in the database contains only the basic data about the company (name, country and values of the company related criteria) as well as the relationships to the carriers and terminal operators. As a consequence, there is no difference among company agents in terms of used negotiation strategies and algorithms for calculation of the best offer that the company agent is capable of making according to the client's criteria. The multi-agent paradigm allows solving this issue by developing a specific agent class for each logistics company agent. To develop a new class with a specific strategy, a subclass of the company agent class has to be implemented and the following behaviors of the company agent can be overloaded in the subclass of a particular company agent:

- The auction strategy must be redefined for the company agent to have another strategy than the current default strategy. It must be programmed how the agent calculates the bid that it is willing to make. For details about the currently implemented default strategies in all auction types see below.

- The calculation method for the most appropriate offer according to the criteria specified by the client. In the default implementation a backtracking search is used to find the most appropriate route and a flat $5 \%$ rate is added to the price as a minimum profit. The utility of this deal is used as the personal evaluation of the deal by a particular company agent. The company can redesign the corresponding behavior to implement any other calculation mechanism.

Currently all agents implement dominant strategies described by M. Wooldridge in [5] for all for auction types in the following way:

- In the English auction, the agent calculates its personal evaluation and if the evaluation is better than the current

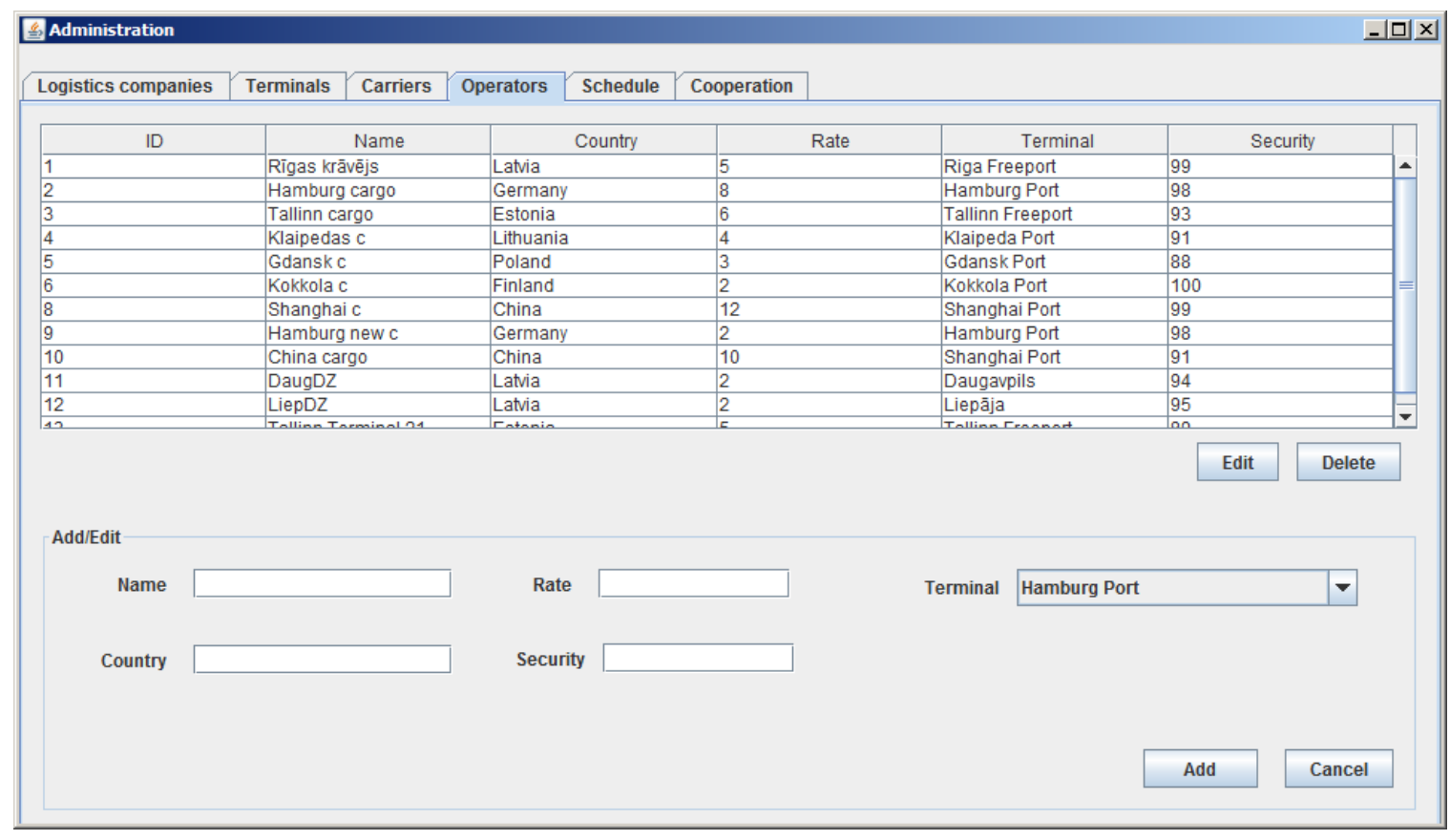

Fig. 3. The administrator's interface. 
- bid, then it makes a bid that improves the utility of the currently best bid by $5 \%$. The price included in the bid is calculated by applying Equation (4).

- In the Dutch auction, the agent waits while the evaluation of the current bid is $10 \%$ worse than its private evaluation and then accepts it.

- In the first-price sealed-bid auction, the agent finds its personal evaluation and increases the price until the evaluation of the deal is $10 \%$ worse than the agent's personal evaluation by applying Equation (4).

- In the Vickery auction, the agent calculates its personal evaluation and bids it.

If the company is willing to employ any other strategy then it has to create its own agent class that implements a particular strategy and uses it to run an agent as an instance of this class.

\section{CONCLUSION AND FUTURE RESEARCH}

The developed tool demonstrates that an electronic marketplace can be created for a particular domain and it is possible to automate negotiations among clients and suppliers. It has been proven by shoving an example in the cargo transportation and logistics domain. Additionally, it is possible to automate not only well-studied single attribute negotiations, but also multi-attribute ones, so finding the best possible deal for each particular client according to his criteria. The proposed evaluation model is developed to be open in the sense that new criteria can be added to the model according to the new trends in the market.

The paper proves the ability of the multi-agent paradigm to support two requirements at the same time, namely the creation of the marketplace where autonomous software components represent their users and the system being available online. The marketplace is open in one more sense. New logistics companies in the person of the corresponding agents can join the marketplace. New agents can be created just by filling in the information about the company.

The main direction of the future research is to actually implement agents with different strategies into the developed marketplace. That would make the market flexible in the sense that it would allow the use of different strategies by company agents. It would be the choice of the company as to which strategy to employ. As M.P. Wellman et al. [13] have outlined, there are very different negotiation strategies even in rather simple domains and agents can have significant improvements in their profit if they find the strategy that works the best against the set of strategies used by their competitors.

\section{ACKNOWLEDGEMENT}

The research has been partly supported by FP7 project eINTERASIA: "ICT Transfer Concept for Adaption, Dissemination and Local Exploitation of European Research Results in Central Asia".

\section{REFERENCES}

[1] Lavendelis, E. and Grundspenkis, J., "Multi-Agent Auction Based Simulation Tool for an Insurance Policy Market," International Scientific
Journal of RTU, Applied Computer Systems, vol. 15, pp. 5-13, 2014. http://dx.doi.org/10.2478/acss-2014-0001

[2] David, E., et al. (eds.), "Agent-Mediated Electronic Commerce. Designing Trading Strategies and Mechanisms for Electronic Markets," AMEC and TADA 2012, Valencia, Spain, June 4th, 2012, Revised Selected Papers. Lecture Notes in Business Information Processing. vol. 136, p. 160, 2013.

[3] Debenham, J. and Simoff, S., "Three Technologies for Automated Trading," Artificial Intelligence in Theory and Practice. IFIP International Federation for Information Processing, vol. 217, pp. 405414, 2006. http://dx.doi.org/10.1007/978-0-387-34747-9_42

[4] Liu, K., "A Model of Protocol for Automated Negotiation System," Proc. of the 2nd Int. Conf. on Green Communications and Networks, GCN 2012, vol. 3, Lecture Notes in Electrical Engineering vol. 225, pp. $407-$ 412, 2013. http://dx.doi.org/10.1007/978-3-642-35470-0 49

[5] Wooldridge, M., An Introduction to MultiAgent Systems, 2nd ed., John Wiley and Sons, p. 484, 2009.

[6] Lavendelis, E. and Grundspenkis, J., "Simulation Tool for Multicriteria Auctions in Transportation and Logistics Domain," Proc. of the Int. Conf. on Computer Systems and Technologies, CompSysTech'06, June 15-16, 2006, Veliko Tarnovo, Bulgaria, ACM Bulgaria, pp. IIIB.17-1 - IIIB.176, 2006.

[7] Grundspenkis, J. and Lavendelis, E., "Multiagent Based Simulation Tool for Transportation and Logistics Decision Support," Proc. of the 3rd Int. Workshop on Computer Supported Activity Coordination, CSAC 2006, Cyprus, Paphos, pp. 45-54, May 16-19, 2006.

[8] Sycara, K., "Problem Restructuring in Negotiation," Management Science, vol. 37, no. 10, Oct. 1991. http://dx.doi.org/10.1287/mnsc.37.10.1248

[9] David, E., Azoulay-Schwartz, R. and Kraus, S., "An English Auction Protocol for Multi-attribute Items," Agent-Mediated Electronic Commerce IV. Designing Mechanisms and Systems. Lecture Notes in Computer Science, vol. 2531, pp. 52-68, 2002.

[10] Bichler, M. and Kalagnanam, J., "Configurable offers and winner determination in multi-attribute auctions," European J. of Operational Research, vol. 160, Issue 2, pp. 380-394, 2005. http://dx.doi.org/10.1016/j.ejor.2003.07.014

[11] Lavendelis, E., "Extending the MASITS Methodology for General Purpose Agent Oriented Software Engineering," Proc. of 7th Int. Conf. on Agents and Artificial Intelligence, ICAART, Lisbon, Portugal, pp. 157165, Jan. 2015. http://dx.doi.org/10.5220/0005202201570165

[12] Bellifemine, F.L., Caire, G. and Greenwood, D., Developing Multi-Agent Systems with JADE. Wiley Series in Agent Technology, p. 300, 2007. http://dx.doi.org/10.1002/9780470058411

[13] Wellman, M.P., Greenwald, A. and Stone, P., Autonomous Bidding Agents: Strategies and Lessons from the Trading Agent Competition. (Intelligent Robotics and Autonomous Agents). The MIT Press, p. 238, 2007, ISBN 026223260X

Egons Lavendelis graduated from the Faculty of Computer Science and Information Technology of Riga Technical University (Riga, Latvia) and received Dr.sc.ing in 2009. The topic of the Doctoral Thesis was "Open Multiagent Architecture and Methodology for Intelligent Tutoring System Development".

He started to work as a Researcher in 2005. Since 2010 he has been a Senior Researcher and since 2013 he has been an Assistant Professor and Senior Researcher at Riga Technical University, Department of Artificial Intelligence and Systems Engineering (former Department of System Theory and Design). His research interests are agent technologies, multi-agent systems, agentoriented software engineering, intelligent tutoring systems and multi-robot systems.

E-mail: egons.lavendelis@ @rtu.lv

Janis Grundspenkis is a Professor at Riga Technical University. He is the Dean of the Faculty of Computer Science and Information Technology and the Head of the Department of Artificial Intelligence and Systems Engineering (former Department of System Theory and Design). He obtained Dr.sc.ing. in 1972, Dr.habil.sc.ing. in 1993 both from Riga Technical University. His research interests are artificial intelligence, agent technologies and computerbased tutoring systems.

$\mathrm{He}$ is a member of the Institute of Electrical and Electronics Engineers (IEEE) and Association for Computing Machinery (ACM). He is a full member of the Latvian Academy of Sciences.

E-mail: janis.grundspenkis@rtu.lv 